\title{
A long period grating based directional flow sensor
}

\author{
Stephen J Buggy, Stephen W James, Ralph P Tatam ${ }^{1}$ \\ Engineering Photonics Group, School of Engineering, Cranfield University, MK43 0AL, UK
}

\begin{abstract}
A chirped long period grating (LPG) is used as a directional flow sensor by monitoring the change in the transmission spectrum upon immersion in an oil of refractive index higher than that of the fibre cladding. The change in profile of an attenuation band is shown to depend on the direction of immersion, indicating the possibility of using such devices in applications such as resin flow sensing in the manufacture of fibre reinforced plastic composite structures.
\end{abstract}

Keywords: Chirped long period grating, directional flow sensor

\section{INTRODUCTION}

The sensitivity of long period gratings (LPGs) to external perturbation has been reported previously and has been exploited for sensing parameters such as temperature, strain and refractive index ${ }^{1}$. The refractive index response of an LPG has been exploited to has been used to demonstrate a liquid level sensor ${ }^{2}$. Khaliq et al demonstrated the use of a uniform period LPG as a liquid level sensor by monitoring the change in the transmission spectrum when the LPG was partially immersed in a liquid of refractive index lower than that of the cladding. The LPG attenuation bands were observed to split as a different phase matched resonant condition existed for the section of the fibre immersed in the liquid as compared to the section in air. The relative transmission depth for the split attenuation bands was shown to be a function of the proportion of the LPG immersed in the liquid. Here the concept of the LPG based liquid level sensor is developed further by demonstrating a sensor which also provides information on the direction of immersion, by exploiting the properties of a chirped LPG.

A chirped long period grating has a periodicity that varies as a function of position along the axial length of the LPG, which results in a broadening of the bands and a decrease in attenuation, compared with that of a uniform period LPG of the same length ${ }^{3}$. The spectrum of a chirped LPG may be explained by considering it as a composite of separate LPGs of differing but closely matched periodicity. A representation of this is shown in figure 1. The separate period LPGs, $\Lambda_{1}, \Lambda_{2}, \Lambda_{3}$, all form attenuation bands of differing central wavelengths. The composite band resulting from the chirped LPG is shown in figure 1 (a) and illustrates the broadening effect on the band. The reduction in attenuation is a result of the dependence of the depth of the attenuation band on the length of the $\mathrm{LPG}^{4}$.



(a)



(b)

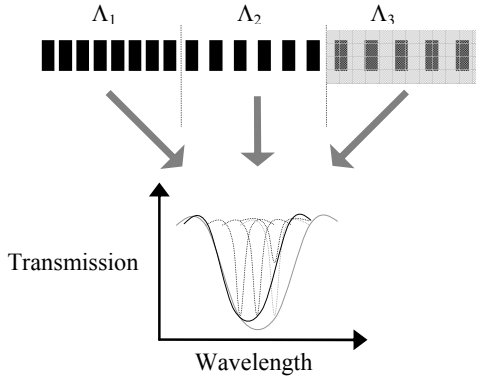

(c)

Figure 1. Simplified representation of a chirped LPG; (a) shows the composite band formed from the three closely matched but different period LPGs, (b) the effect of covering the section of the LPG with a period of $\Lambda_{1}$, (c) the effect of covering the section of the LPG with a period of $\Lambda_{3}$.

\footnotetext{
${ }^{1}$ r.p.tatam@cranfield.ac.uk; phone: +44 (0)1234 754630; fax: +44 (0)1234 750728; www.cranfield.ac.uk/soe/cpoe 
If a section of the LPG is covered, e.g. $\Lambda_{1}$ of figure 1 (b), with an oil of refractive index higher than that of the cladding, the composite band period experiences a slight decrease in attenuation and a positive shift of the lower wavelength edge (because, when exposed to a refractive index higher than that of the cladding, the LPG has low sensitivity to the surrounding refractive index, the wavelength shift may be neglected). This may be explained by the coupling to leaky cladding modes ${ }^{5}$, which results in a decrease in the extinction ratio of the attenuation band corresponding to section $\Lambda_{1}$. The expected result for covering section $\Lambda_{3}$ is shown in figure 1 (c). This demonstrates the potential for the use of such devices as directional flow sensors. The discussion is concerned with the refractive indices higher than that of the cladding, as this is representative of the refractive index of resin systems used for structural components.

\section{EXPERIMENT}

An LPG of length $40 \mathrm{~mm}$ was fabricated in hydrogen loaded single mode fibre of cut of wavelength 650nm (Fibercore SM750). The fibre was placed behind a Vernier calliper with a fixed slit width of $200 \mu \mathrm{m}$ that was illuminated by a UV laser beam at a wavelength of $266 \mathrm{~nm}$, provided by a frequency quadrupled Nd:YAG laser. A computer controlled translation stage was used to move the fibre behind the slit to create a linearly chirped period, from $395 \mu \mathrm{m}$ to $405 \mu \mathrm{m}$. The LPG was subsequently annealed at $100^{\circ} \mathrm{C}$ for 24 hours to ensure that the hydrogen had diffused out and that the spectrum was stable 6 .



(a)

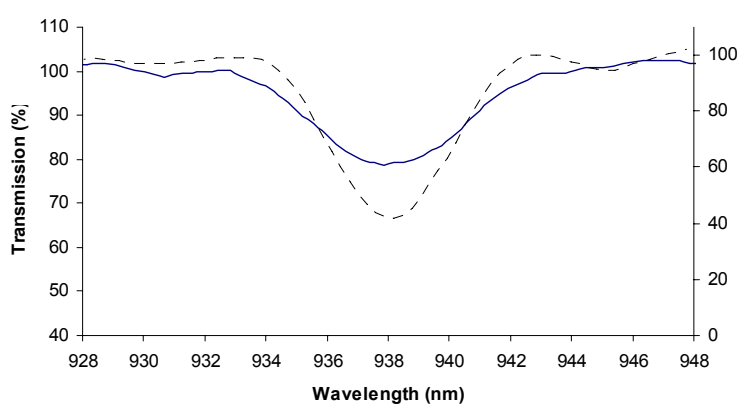

(b)

Figure 2. Transmission spectrum of a chirped LPG of length $40 \mathrm{~mm}$ and linearly chirped period $395 \mu \mathrm{m}-405 \mu \mathrm{m}$, fabricated in Fibercore SM750. Band 6 is highlighted and shown compared with a band in the same spectral location (b) of an LPG of uniform period $400 \mu \mathrm{m}$ (dashed).

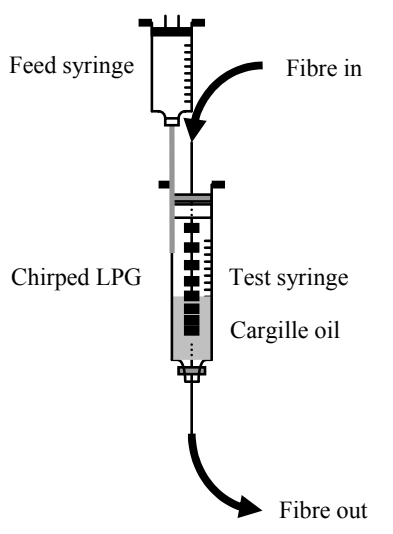

(a)



(b)

Figure 3. Experimental setup for chirped long period grating flow sensor (a) orientation of the chirped LPG for the flow sensing experiments (b).

The transmission spectrum of the chirped LPG, shown in figure 2, was monitored by coupling the output from a tungsten-halogen white light source into the fibre and coupling the transmitted light to a CCD spectrometer (OceanOptics S2000). The experimental setup is shown in figure 3 (a). A feed syringe allowed a test syringe, graduated 
with $0.01 \mathrm{ml}$ marks, to be gradually filled with a Cargille refractive index oil of $1.56 \pm 0.0002$. The fibre was held in place with rubber grommets to prevent the fibre from bending, which is known to distort the transmission spectrum ${ }^{8}$. The rubber grommets also provided a seal to ensure that there was no loss of liquid. The needle from the feed syringe was placed close to the wall of the test syringe to ensure there was no contact during feeds between oil and LPG before the oil reached the bottom of the test syringe. The experiment was repeated with the orientation of the chirp reversed to simulate the effect of the liquid flowing over the LPG from the opposite direction, as illustrated in figure 3 (b).

\section{RESULTS}

As the test syringe was filled with the oil, the transmission spectrum of the LPG was recorded for each $2.7 \mathrm{~mm}$ increment in the depth of the oil. Figure 4 shows the response of band 6 to the gradual covering of the LPG by the oil for the different test orientations. With the LPG oriented with decreasing period relative to the oil, experiment 1, (figure 4(a)), an apparent negative wavelength shift is measured. This is caused by a reduction of the extinction at longer wavelengths due to the cladding/oil structure of the covered section no longer supporting propagating cladding modes. The result for experiment 2 is shown in figure 4 (b).

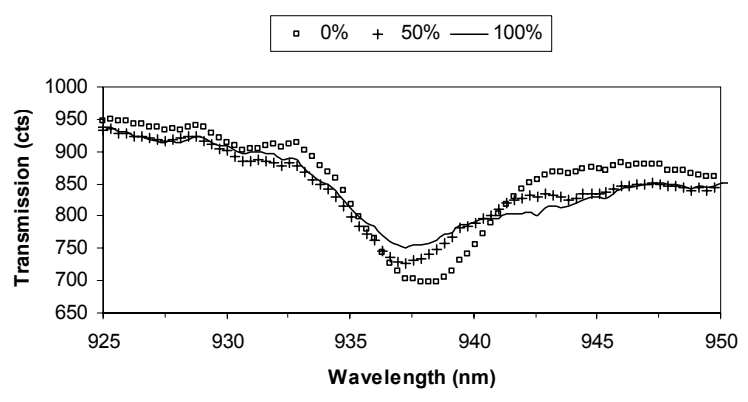

(a)

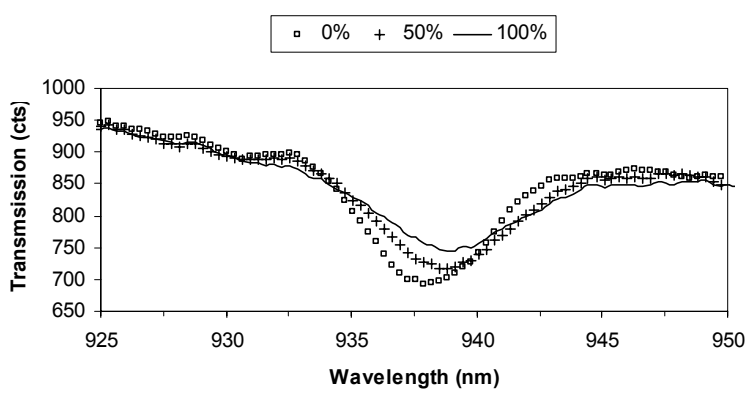

(b)

Figure 4. Change in shape of band 6 for gradual immersion in 1.56 Cargille index oil. Experiment 1 (a) and experiment 2 (b). $0 \%$ is no coverage, $50 \%$ half and $100 \%$ total coverage.

Figures 5 (a) and (b) show the wavelength shift of the central wavelength of attenuation band 6 and the minimum transmission value at that wavelength for the different test orientations. The band minimum was calculated by fitting a $6^{\text {th }}$ order polynomial to the spectra. For both experiments, an apparent wavelength shift of $\sim 1 \mathrm{~nm}$ is observed, with directional information given by the direction of shift. In experiment 1 (figure 5 (a)) the wavelength shift is negative while experiment 2, (figure 5 (a)) the wavelength shift is positive. The transmission change is similar (10\%) for both orientations.

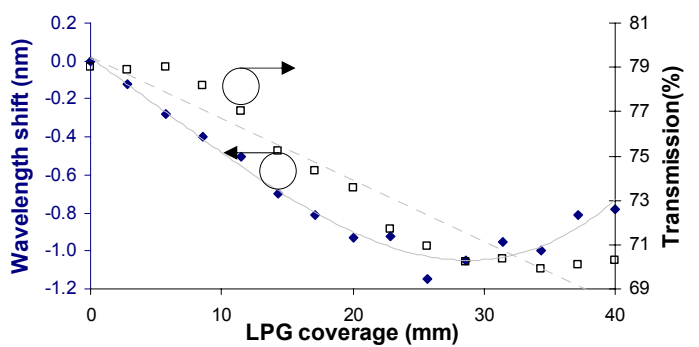

(a)

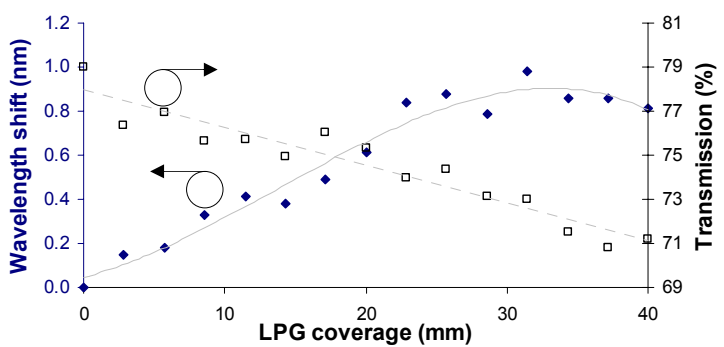

(b)

Figure 5. Wavelength shift ( $\downarrow)$ of band 6 and minimum transmission value ( $\square$ ) against LPG coverage with Cargille index oil (1.56) for LPG arranged with decreasing period (a) and increasing period (b). Grey lines are shown only as a visual aid. 


\section{DISCUSSION}

The results presented in figures 4 and 5 show the wavelength shift and change in transmission of band 6 for the gradual immersion of the grating in the Cargille oil. The apparent wavelength shift of the band is shown to be dependant on the direction of flow of the oil surrounding the fibre. The change in the extinction of the band is shown to have no directional dependency. When the oil covers $75 \%$ of the LPG the results for both orientations of the LPG indicate a change in direction for wavelength shift as the coupling to leaky cladding modes begins to dominate. When the LPG is fully immersed in the high index oil the cladding/oil structure no longer supports propagating cladding modes but leaky cladding modes exist due to Fresnel reflections from the cladding oil interface. Coupling to such modes is weak when the index difference between the cladding and oil is small and results in attenuation bands with a poor extinction ratio. This effect and the broad bands exhibited by chirped LPGs may contribute to errors in determining the wavelength of the band minimum.

\section{CONCLUSION}

A directional flow sensor using a chirped LPG is demonstrated with an oil of refractive index higher than that of the silica fibre,. An attenuation band is shown to have directional dependence on immersion in the oil leading to the possibility of a flow sensor with directional sensing capability. As epoxy resin used in the fabrication of composite structural components typically have a refractive index in the range $1.5-1.6$, the results indicate the potential for the use of chirped LPGs as directional flow sensors to monitor the infusion of resins into reinforcing fibre layups.

The authors gratefully acknowledge the support for this work from the Engineering and Physical Sciences Research Council (EPSRC), UK.

\section{REFERENCES}

1 S. W. James and R. P. Tatam "Optical Fibre Long Period Grating Sensors: Characteristics and Application," Measurement Science and Technology 14, 49-61 (2003).

2 S. Khaliq, S. W. James and R. P. Tatam, "Fibre optic liquid level sensor using a long period grating," Optics Letters 6, 1224-6 (2001).

3 Z. Wu, Z. Dong, and Z. Xu, "The chirp characteristics of long-period fiber gratings" Proc. SPIE 4595, 89-93 (2001).

$4 \quad$ R. Kashyap, Fibre Bragg Gratings, chapter 4, Academic, New York (1999).

5 H. J. Patrick, A. D. Kersey and F. Bucholtz, "Analysis of the response of long period fibre gratings to external index of refraction," Journal of Lightwave Technology 16, 1606-12 (1998).

6 K. Fujita et al, "Dynamic evolution of the spectrum of long-period fiber Bragg gratings fabricated from hydrogen-loaded optical fiber by ultraviolet laser irradiation," Applied Optics 44, 7032-8 (2005).

7 O. Duhem, J-F. Henninot, M. Warenghem and M. Douay, "Demonstration of long-period grating efficient coupling with an external medium of a refractive index higher than that of silica," Applied Optics 37, 7223-8 (1998).

8 C. C. Ye, S. W. James and R. P. Tatam, "Simultaneous temperature and bend sensing using long-period fibre gratings," Optics Letters 25, 1007-9 (2000). 